\title{
Impacto económico, valoración de evento y recuerdo de patrocinio en un maratón popular
}

\author{
Economic impact, event valuation and sponsor recall in a popular marathon
Benito Pérez-González ${ }^{1}$ José Luis Gómez-Carmona², Jairo León-Quismondo ${ }^{3}$, Pablo Burillo $^{3}$, Rosario Teva-Villén ${ }^{4}$, Álvaro Fernández-Luna ${ }^{3}$

1 Facultad de Economía y Comunicación. Universidad Internacional de la Rioja. España.

2 Facultad de Ciencias de la Actividad Física y del Deporte. Universidad de León. España.

3 Facultad de Ciencias de la Actividad Física y el Deporte. Universidad Europea de Madrid. España.

4 Departamento de Deporte e Informática. Universidad Pablo de Olavide. España.

CORRESPONDENCIA:

Benito Pérez-González

benitoperezgonzalez@gmail.com

Recepción: octubre 2020 • Aceptación: mayo 2021
CÓMO CITAR EL ARTÍCULO:

Pérez-González, B., Gómez-Carmona, J. L., León-Quismondo, J., Burillo, P., Teva-Villén, R., \& Fernández-Luna, Á. (2021). Impacto económico, valoración de evento y recuerdo de patrocinio en un maratón popular. Cultura, Ciencia y Deporte, 16(50), 617-630. http://dx.doi.org/10.12800/ccd.v16i50.1616

\section{Resumen}

El objetivo de este estudio fue medir el impacto económico, la satisfacción, el impacto turístico y el retorno de objetivos de patrocinio de las marcas colaboradoras en un maratón celebrado en 2019 en una ciudad española de cerca de 200.000 habitantes. El evento incluyó las pruebas de maratón, media maratón y maratón por relevos. Se realizó un total de 293 encuestas de un total de 1276 inscritos. La mayor parte de estos residían en la ciudad del evento $(58 \%, n=740)$, en otros puntos de España $(40 \%, n=510)$ y un grupo más pequeño $(2 \%)$ en el extranjero $(n=26)$. El impacto directo se midió utilizando la metodología de análisis coste beneficio (ACB) y alcanzó la cifra de 134,057.42 €. Adicionalmente se evaluó el impacto indirecto utilizando tablas Input-Output obteniendo una estimación de 73,866.83 €. Para el análisis del impacto turístico y satisfacción se aplicaron estadísticos descriptivos y test no paramétricos de comparación de medias. No se observaron diferencias significativas en la mayoría de variables, con valoraciones elevadas en reconocimiento promocional de la ciudad del evento, orgullo, turismo y vinculación de dicha ciudad con el deporte. Finalmente, el recuerdo de patrocinio de las marcas participantes es superior en los recuerdos inducidos respecto a los espontáneos, salvo en el caso del patrocinador principal, con elevado recuerdo en ambos tipos de recuerdo.

Palabras clave: maratón, eventos deportivos, impacto económico, recuerdo de marca, patrocinio deportivo.

\section{Abstract}

This study aimed to measure the economic impact, satisfaction, tourist impact, and return on sponsorship objectives of the partner brands in a Marathon held in 2019 in a city of about 200,000 inhabitants. The event included the marathon, half marathon, and relay marathon races. A total of 293 surveys were conducted out of a total of 1167 participants. Most of the registered participants lived the city of the event $(58 \%, n=740)$, other parts of Spain $(40 \%, n=510)$, and a smaller group ( $2 \%)$ from abroad $(n=26)$. The direct impact was measured using the cost-benefit analysis methodology (CBA) and reached the amount of $€ 134,057.42$. Additionally, the indirect impact was evaluated using Input-Output tables, obtaining an estimate of $€ 73,866.83$. For the analysis of tourist impact and satisfaction, descriptive statistics and nonparametric tests for means comparison were applied. We did not observe significant differences in most of the analyzed variables, but we found high valuations in promotional recognition, pride, tourism, and city's link with sports. Finally, the sponsorship recall of the participating brands is higher in the induced recall compared to the spontaneous, except in the case of the main sponsor, with high recall in both types of recall.

Key words: marathon, sport events, economic impact, brand recall, sponsorship. 


\section{Introducción}

Los eventos deportivos generan efectos positivos en la población, región o país donde tienen lugar. Estos efectos, según Fernández y Alonso (2012), pueden verse reflejados en el incremento de ingresos económicos en los territorios de implantación, la construcción de instalaciones e infraestructuras que mejoran la funcionalidad y estética de las localizaciones $\mathrm{y}$, finalmente, en la promoción de las localidades a través del efecto mediático, constituido a razón de los medios de comunicación y los propios asistentes.

El Anuario de Estadísticas Deportivas en España (Ministerio de Cultura y Deporte, 2020) refleja que la segunda modalidad deportiva más practicada es la carrera a pie ( $10.6 \%$ de los encuestados), por detrás únicamente de la gimnasia de mantenimiento (19.2\%). El running es precisamente la actividad que muestra el mayor crecimiento porcentual entre 2010 y 2015 , habiendo pasado de un $4.8 \%$ al $10.6 \%$ (Felipe et al., 2018). Estas cifras han facilitado el auge de las carreras populares en España, donde se celebran más de 3000 eventos de este tipo de manera anual (Burillo \& Pérez-González, 2016). Ante tal número de oferta de carreras de resistencia y la magnitud que están adquiriendo en volumen de participantes, la gestión de estos eventos es cada vez más profesionalizada. Los maratones son los eventos que se posicionan como referentes a nivel internacional, siendo además las más complejas en términos de organización. Hasta ahora, las principales líneas de investigación científica de este tipo de carreras se han llevado a cabo desde el punto de vista fisiológico o psicológico para determinar las variables que afectan al rendimiento. Por su parte, las publicaciones relacionadas con la gestión de estos eventos, han centrado su interés en temas como las motivaciones de los corredores (Clough et al., 1989; Duclos-Bastías et al., 2021; Funk et al., 2007), las diferencias en los motivos en función del género (Ogles \& Masters, 1995; Salguero \& Martos, 2011), o la segmentación de participantes en función de sus perfiles (Martínez-Cevallos et al., 2020b; Parra-Camacho et al., 2020a).

Indudablemente, la celebración de este tipo de eventos tiene una gran influencia en las localidades por las que discurren, generando un impacto económico significativo en el área (Cobb \& Olberding, 2007; Saaymann \& Saayman, 2012). Estos datos son de gran utilidad para la toma de decisiones relativas a la continuidad y para la inversión de empresas en estas localizaciones. Profundizando en el impacto económico, este se define como el cambio económico neto en los ingresos de los anfitriones de eventos que resultan del gasto atribuido a los visitantes. Estos son empleados para subsidiar eventos turísticos que a su vez movilizan promociones, cambios estructurales y estéticos que intrínsecamente también son de atracción para más visitantes.

Estas visitas suscitan un gasto de dinero en las localidades que generan ingresos y empleos para los residentes, completando un círculo virtuoso de desarrollo económico (Crompton, 2006). El impacto económico de eventos puede ser tipificado en dos modalidades:

- Impacto directo: es el generado por el gasto por los visitantes del evento en los distintos sectores económicos.

- Impacto secundario: se corresponde con el efecto dominó provocado por las rondas adicionales de recirculación de la inyección de gasto inicial de los visitantes. Este impacto a su vez puede ser de dos tipos, indirectos e inducidos (Jago \& Dwyer, 2006).

Los informes que establecen mediciones de los impactos económicos de eventos deportivos vinculados a ciudades, generalmente con clara asociación con el turismo, resultan de gran trascendencia y necesidad para poder establecer una óptima estimación de su repercusión (Blanco-Gregory, 2018). Estos impactos se producen principalmente en grandes eventos, pero también en eventos de menor envergadura, los cuales igualmente generan un impacto positivo, tanto a nivel económico (Agha \& Rascher, 2016; Baños et al., 2012; Murillo et cl., 2016) como social (Parra-Camacho et al., 2020b).

Existen diferentes metodologías para cuantificar el impacto económico. Entre ellas, podemos destacar, el Análisis Coste Beneficio (ACB) y las tablas Input-Output (Fernández \& Alonso, 2012). El primero se fundamenta en la evaluación de las implicaciones de índole socioeconómico de eventos a través de la estimación del valor actual neto VAN en función del análisis de costes y beneficios. El segundo y más utilizado se basa en el establecimiento de interrelaciones entre las variables monetarias relacionadas con el sector de análisis y la propia economía.

En España, al igual que en otros países, el maratón es la distancia de referencia para la celebración de eventos deportivos de gran magnitud. Los circuitos de Valencia, Barcelona, Sevilla y Madrid son pruebas consolidadas y las de mayor importancia, por prestigio, magnitud y número de corredores. En la Tabla 1 pueden observarse de manera somera los datos de impacto y gasto por participante de las últimas competiciones de maratón en diferentes ciudades españolas. 
Tabla 1. Impacto económico de los maratones españoles (elaboración propia).

\begin{tabular}{|c|c|c|c|c|c|c|c|}
\hline Maratón & Participantes & $\begin{array}{l}\text { Impacto económico } \\
\text {-gasto turístico }\end{array}$ & $\begin{array}{l}\text { Impacto económico } \\
\text { total economía local }\end{array}$ & $\begin{array}{c}\text { Gasto por } \\
\text { participante }\end{array}$ & Año & Fuente & Retorno \\
\hline Valencia & 25,000 & $22,803,199$ & $52,052,098$ & 960.41 & 2020 & (Maudos et al., 2020) & 3.7 \\
\hline Sevilla & 13,000 & $14,000,000$ & & 1021.89 & 2020 & $\begin{array}{l}\text { (Zurich Maratón de Sevilla, } \\
\text { 2020) }\end{array}$ & \\
\hline Madrid & 35,000 & & $42,915,052$ & & 2019 & (Europa Press, 2019) & 22.9 \\
\hline Málaga & 13,160 & $9,400,000$ & & & 2016 & $\begin{array}{l}\text { (Andalucía Información, } \\
\text { 2016) }\end{array}$ & \\
\hline Gran Canaria & 1398 & $3,000,000$ & & 2145.92 & 2017 & (RTVC, 2017) & \\
\hline Castellón & 3000 & $1,600,000$ & & 418.19 & 2017 & (COPE, 2017) & \\
\hline Bilbao & 13,400 & $4,000,000$ & & 298.50 & 2019 & (El Correo, 2019) & \\
\hline Lanzarote & 1441 & 665,000 & & 461.48 & 2015 & (Lancelot Digital, 2015) & 7.49 \\
\hline Ibiza & 2107 & $1,952,633$ & & 968.08 & 2018 & (Sánchez-Venzalá, 2019) & \\
\hline Vitoria & 4475 & $2,039,660$ & & 372.54 & 2016 & (42K Running, 2016) & 58.8 \\
\hline Tarragona & 1600 & 200,000 & & 125 & 2017 & (Aguilera, 2017) & \\
\hline
\end{tabular}

Según Murua (2013), realizar una interpretación rigurosa acerca del impacto generado por la celebración de los distintos maratones en el panorama nacional no es tarea sencilla por la escasez de estudios realizados bajo directrices científicas y por el difícil acceso a la información (solo el informe económico del maratón de Valencia se encuentra disponible públicamente). En la elaboración de la Tabla 1, a excepción de Valencia, se han tomado como consideración referencias en prensa digital, con cifras que en algún caso son aproximaciones más que aseveraciones certeras y fidedignas. Por ello, la interpretación que se haga del conjunto tendrá un talante orientador con una consideración más cualitativa que cuantitativa. Sería interesante poder analizar los métodos de realización de los estudios para certificar el motivo de tal dispersión, además de especificar el impacto económico si es de gasto turístico o de impacto sobre el total de economía local, esto es, impacto directo o indirecto (Salgado-Barandela et al., 2013). Asimismo, la interpretación de las cifras en algunas carreras debe ser tomada con cautela, añadiendo también cierto grado de distorsión el hecho de que en muchas competiciones se incluyen pruebas con menores distancias que pueden desfigurar la pureza considerativa del maratón como generador inequívoco de impacto económico.

Los eventos deportivos como el maratón no solo provocan un impacto económico, sino que consiguen beneficios sociales, culturales, la mejora de la percepción de la ciudad donde se organiza, la dinamización social o la vinculación de la ciudad con el deporte (Coleman \& Ramchandani, 2010; Fourie \& Santana-Gallego, 2011;
Bosch et al., 2019). La actividad físico-deportiva, por su gran vinculación social y arraigo en la población, es un importante sector con elevadas implicaciones económicas. Igualmente, el turismo desarrolla una acción económica de primer nivel. Por tanto, es lógico suponer que la dualidad generada entre deporte y turismo, a través de los eventos deportivos, tendrá una importante repercusión social y económica y una relevante proyección. Si este ámbito lo extrapolamos al entorno municipal, se pueden entender las importantes influencias que los eventos deportivos como formas turísticas tienen no solo para las economías, sino también para la imagen reputacional de la ciudad (Felipe et al., 2015).

En esta línea es necesario aludir a la marca ciudad, de la que hay que partir de la consideración de ciudad como sujeto referencia predominante de los eventos deportivos, que además se entiende como un producto con una fusión de atributos tangibles (edificios, parques, carreteras, etc.) e intangibles (valores, cultura, imagen, etc.), susceptibles de valoración y generación de un determinado nivel de satisfacción. De esta fusión surge la concreción de unas características específicas de la ciudad que permitirá la diferenciación con respecto a otras ciudades junto con su imagen (Kaplanidou et al., 2012), y al lado de esta, su marca e imagen de marca. Esta marca ciudad supone un activo intangible que reportará gran atractivo, valoración a la ciudad, favoreciendo que los participantes recomienden su visita (Martínez-Cevallos et al., 2020a). Como tal, la imagen de marca no debe ser abandonada a su construcción azarosa, sino que debería estar integrada dentro de un plan de marketing estratégico, donde la 
transmisión de una imagen de fortaleza, diferenciación, reconocimiento y, ante todo, susceptible de opinión favorable, debería estar planificada (Sáez-Vegas et al., 2011). Igualmente, la gestión del evento influye en la satisfacción final de los asistentes. Por ello, estudios recientes, además de las variables relacionadas con la imagen del destino y las intenciones futuras, incluyen elementos relacionados con la calidad de la experiencia, como el personal, los servicios y las instalaciones (Burillo et al., 2018; Calabuig-Moreno et al., 2016; Vegara-Ferri et al., 2020).

Ante esta perspectiva, la organización de eventos deportivos con visionado tanto presencial como a distancia a través de los medios de comunicación, cada vez más diversificados, globalizados y democráticos, es un aspecto clave para ayudar en la generación de la marca ciudad y que debería estar inmersa en las estrategias de marketing de las ciudades. Consecuentemente, la atracción turística posterior al evento en sí se verá positivamente afectada, generando una clara amplificación de los influjos del turismo deportivo con sus firmes implicaciones en cuanto a impactos económicos (Blanco-Gregory, 2018). Estas circunstancias están presentes en ciudades como Barcelona, Málaga, Rotterdam, Helsinki o Turín (Fourie \& Santana-Gallego, 2011; Gibson et al., 2008), las cuales han maximizado su potencial activamente a través de la conjunción del deporte y las acciones de marketing de la ciudad (Berg, 2002; citado en Sánchez-Fernández \& RodríguezCampo, 2012). Así pues, el turista deportivo va construyendo un sentimiento de pertenencia con la ciudad que perdura en el tiempo, lo que refuerza un lazo de complicidad afectiva. Esta circunstancia permite que la imagen y la marca ciudad se forje y se transmita a través de múltiples agradecidos embajadores que han bautizado las calles con el sudor de sus gestas (Theodorakis et al., 2015).

Finalmente, un elemento clave en los eventos de maratón es el patrocinio, como elemento imprescindible de promoción para captar la atención del público y asociar los valores de su marca a los del deporte patrocinado. En el ámbito de la eficacia publicitaria, el recuerdo de la marca se ha establecido como uno de los principales criterios de medida. En ese sentido, Paz et al. (2000) describen el post-test en función del recuerdo como una de las principales técnicas del post-test publicitario, pudiendo ser este espontáneo o inducido. Concretamente, en lo referente a las estrategias de patrocinio deportivo, Banks (1992) sugiere el recuerdo como un indicador efectivo para las empresas en la asignación de recursos al patrocinio.

El incremento de patrocinio eventos de running en España creció hasta 800 acuerdos de patrocinio, con una variedad de 400 marcas en 2019 (CMD Sport, 2019). Por ello, se hace necesario que las marcas conozcan además del retorno de inversión, el retorno de objetivos de patrocinio que justifica las grandes sumas depositadas en este tipo de eventos.

Por todo lo expuesto anteriormente, no tenemos evidencia de un estudio que analice los eventos de maratón aplicando metodología científica y teniendo en cuenta una visión holística que incluya el impacto social, económico y de las marcas patrocinadoras. Por ello, consideramos importante llevar a cabo un estudio integral, que aúne la perspectiva de impacto socioeconómico y de patrocinio de un evento deportivo popular como es el de esta prueba, estableciendo los siguientes objetivos:

1. Medir el impacto económico directo del Campofrío Maratón 2019 Burgos. Para ello se evaluó el gasto realizado, por los participantes y acompañantes de fuera de Burgos, durante los días de estancia en la ciudad.

2. Medir el impacto económico indirecto del Campofrío Maratón 2019 Burgos.

3. Evaluar el retorno de objetivos de patrocinio de las marcas colaboradoras a través del recuerdo espontáneo y el recuerdo inducido que tenían los participantes de los patrocinadores de esta prueba atlética.

4. Conocer la satisfacción sobre el evento, así como el impacto social y turístico percibido por los atletas participantes.

\section{Método}

Instrumentos

Para el trabajo de investigación se diseñó un cuestionario ad-hoc. Se incluyeron preguntas para los participantes adultos de las diferentes pruebas ofertadas por los organizadores del evento. El cuestionario utilizado fue adaptado de otros similares utilizados en estudios de impacto económico (Sánchez-Fernández et al., 2016), de impacto social y satisfacción de eventos (Añó et al., 2012) y de recuerdo de marca en eventos deportivos (Pérez-Acosta, 2006). Dicho cuestionario fue subido a la plataforma de cuestionarios de Google, que permite el acceso simultáneo de varios usuarios a un mismo formulario. De igual manera, la base de formularios de Google permite el almacenamiento y tratamiento ordenado de los datos de las diferentes preguntas, permitiendo realizar preguntas de clasificación, de respuesta múltiple, de escala Likert, de respuesta escrita, tanto larga como corta, así como abrir secciones diferentes en función de la respuesta 
a alguna de las preguntas emitidas. El cuestionario se encuentra disponible en el siguiente link: https:// forms.gle/nbMGXz1VwMNqihAj8. Del mismo modo, adjuntamos en el anexo 1 una copia de este.

Una vez diseñado el cuestionario fue presentado a un grupo de expertos, que realizó propuestas de cambios y mejoras sobre el mismo, consiguiendo así una mayor adecuación a los objetivos descritos anteriormente.

Por las características del evento, así como por las exigencias metodológicas y funcionales (Li \& Jago, 2013), para el presente estudio del impacto económico de la prueba, se opta por el análisis de coste y beneficio (ACB) ya que, por su flexibilidad, amplitud y características, es uno de los enfoques más adecuados para la valoración de eventos deportivos como el que nos ocupa (Sánchez-Fernández et al., 2016). En la adaptación de esta metodología se investigan los consumos realizados por participantes del Maratón de Burgos que procediesen de otras localidades. Es importante destacar la involucración no solo de los deportistas participantes en la generación de impacto económico, sino que también se han de considerar a los turistas pasivos, bien sean acompañantes de los primeros o no, ya que al margen de las repercusiones culturales, afectivas y perceptivas, su peso en los impactos económicos de eventos no han de ser olvidados (Hallmann et al., 2011).

El cuestionario final consultó de manera desglosada por el recuerdo de gastos en los siguientes conceptos: alojamiento, restauración, desplazamiento, compras de material deportivo, otras compras, visitas y museos, así como otros gastos. El gasto de inscripción se asoció directamente a cada uno de los encuestados en función de la prueba en la que participaban. La información sobre la inscripción la recibimos directamente de los organizadores del evento.

De manera adicional, se realizó un cálculo del impacto económico indirecto. El impacto indirecto se deriva del impacto directo (Salgado-Barandela et al., 2013). Para medirlo es necesario aplicar un multiplicador sobre el impacto directo obteniendo así el efecto indirecto sobre la economía local. Es lo que se conoce también como el efecto de arrastre que se produce entre las distintas ramas de actividad de una economía.

El multiplicador reconoce el efecto que tiene la actividad económica originada por los visitantes de un evento en el resto de sectores económicos, creando un efecto indirecto en la economía (Crompton, 1995). Los multiplicadores se obtienen a partir de las tablas Input-Output. Dichas tablas desagregan la economía en distintos sectores de la industria examinando los flujos de bienes y servicios entre ellos. El proceso permite obtener un multiplicador separado que debe aplicarse a cada uno de los sectores industriales afectados por el gasto directo inicial. En nuestro caso utilizamos las últimas tablas Input-Output disponibles en la Junta de Castilla y León (2019).

Para la impacto social y satisfacción de eventos incluimos en el cuestionario 8 ítems, arrojando un alfa de Cronbach de 0,628, para que los encuestados los valorasen en una escala Likert de 1 a 5 , siendo 1 : totalmente en desacuerdo; 2: en desacuerdo; 3: indiferente; 4: de acuerdo; 5: muy de acuerdo. Los ítems en cuestión se basaron en los evaluados por Añó et al. (2012) $\mathrm{y}$ fueron los siguientes:

1. Aumenta el reconocimiento y promoción de la ciudad.

2. Representa un elemento de orgullo para la ciudad.

3. Altera el ritmo habitual y perjudica otras actividades.

4. Provoca un incremento del turismo durante y después del evento.

5. Aumenta el empleo.

6. El gasto público es necesario para la celebración de este tipo de eventos.

7. Hace que mucha gente quiera volver a la ciudad para conocerla mejor.

8. Es una oportunidad para aumentar la marca de Burgos como ciudad relacionada con el deporte.

Por último, el cuestionario incluía un apartado para estudiar el recuerdo de patrocinio espontáneo e inducido (Pérez-Acosta, 2006). Para el recuerdo espontáneo se incluía una pregunta para que los encuestados mencionasen marcas patrocinadoras del evento que rememoraran en ese momento. Tras responder, se les mencionaban 12 marcas, algunas de las cuales eran patrocinadoras del Maratón de Burgos y otras no. Los encuestados debían valorar si dichas marcas eran patrocinadoras o no del evento, en una escala Likert de 1 a 5, siendo 1: sí, seguro; 2: creo que sí; 3: no lo sé; 4: creo que no; 5: no, seguro.

Igualmente, en el instrumento final se incluyeron las siguientes variables sociodemográficas: género, grupos de edad basados en otras investigaciones en eventos de running de España (Felipe et al., 2018) y modalidad de participación. La Figura 1 muestra los componentes utilizados en el instrumento.

\section{Universo y muestra de encuestados}

Para la toma de datos, un equipo de 8 personas de la Universidad Isabel I, estuvo presente en el primer día de la recogida de dorsales (12 de octubre de 2019). Todos los encuestadores recibieron una formación previa de 2 horas de duración, impartida por el investigador principal el día anterior de la recogida de dorsales. En 


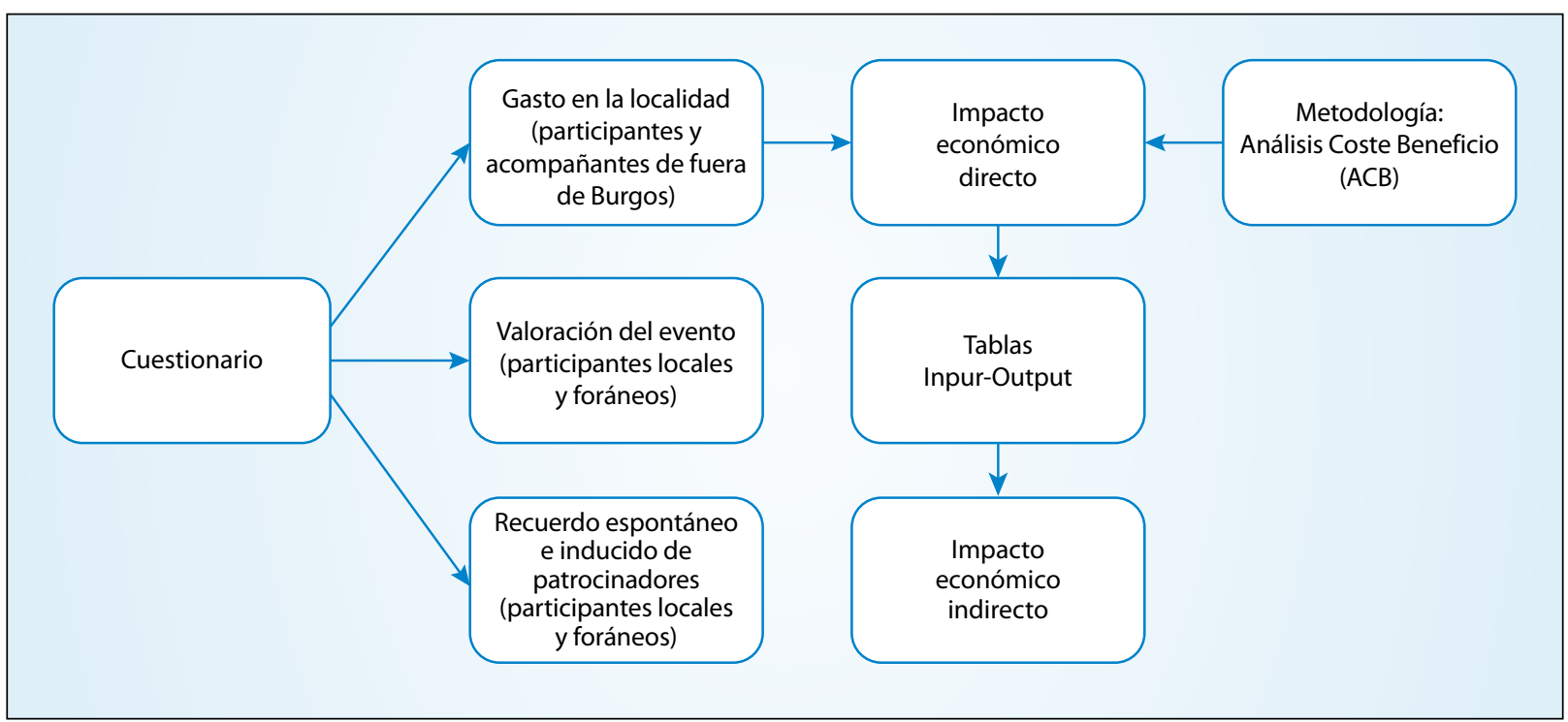

Figura 1. Resumen de instrumentos utilizados.

ella, se les expuso el objetivo principal de la investigación, se les mostró el cuestionario y se les explicó que serían los encargados de encuestar a los participantes que asistiesen a la recogida de dorsales y que voluntariamente quisiesen responder a las preguntas. Todos los encuestadores finalizaron la formación comprendiendo satisfactoriamente el procedimiento y habiendo sido resueltas todas sus dudas. Los encuestadores eran responsables de rellenar el cuestionario digital con las respuestas que recibiesen de cada participante entrevistado, por lo que no se trataba de un cuestionario autoadministrado, sino que contaba siempre con la presencia de un encuestador. La organización también permitía la recogida de dorsales en el día de la prueba (13 de octubre de 2019), pero solo durante una hora, por lo que la mayor parte de participantes $(87 \%)$ $(\mathrm{n}=257)$ se acercaron al punto de recogida el sábado 12 de octubre de 2019.

Para el trabajo de campo se contó con el permiso y colaboración de los organizadores del evento. La realización de encuestas comenzó a las 10 horas y finalizó a las 19 horas, consiguiendo 293 entrevistas sobre un total de 1276 inscritos, de los que finalmente no recogieron su dorsal 109. Los niveles de confianza y margen de error se muestran en las Tablas 2 y 3 .

Referente a las características de la muestra, el $85 \%$ de los participantes fueron de género masculino $(n=249), 75 \%$ eran corredores de media maratón $(n=220), 18 \%$ maratonianos $(n=53)$ y el $6 \%$ participantes en relevos $(\mathrm{n}=7)$. Respecto al origen de los corredores encuestados, $63 \%(\mathrm{n}=183)$ fueron locales, $36 \%$ provenían de otros municipios de España (108), y solo un $1 \%(\mathrm{n}=2)$ fueron corredores extranjeros. Estos datos no difieren mucho del total de inscritos
Tabla 2. Ficha técnica de la encuesta sobre gasto en Burgos. Corredores de fuera de Burgos (objetivos 1 y 2).

\begin{tabular}{lc}
\hline Inscritos totales de fuera de Burgos: & 532 \\
Estimación de corredores que no recogieron el dorsal: & $(82)$ \\
Participantes de fuera de Burgos: & 450 \\
Encuestados totales de fuera de Burgos: & $108(24 \%)$ \\
Nivel de confianza: & $95 \%$ \\
Margen de error: & $8.23 \%$ \\
\hline
\end{tabular}

Tabla 3. Ficha técnica de la encuesta sobre gasto en Burgos. Corredores totales (objetivos 1 y 2 ).

\begin{tabular}{lc}
\hline Inscritos totales: & 1275 \\
Corredores que no recogieron el dorsal: & $(109)$ \\
Participantes finales: & 1167 \\
Encuestados totales: & $293(25.1 \%)$ \\
Nivel de confianza: & $95 \%$ \\
Margen de error: & $4.96 \%$ \\
\hline
\end{tabular}

siendo estos un 58\% ( $n=743$ ) residentes en Burgos, un $40 \%(n=508)$ residentes en otros municipios y un $2 \%$ $(n=24)$ residentes en el extranjero.

\section{Análisis estadístico}

Los datos obtenidos se organizaron en una base de datos del paquete estadístico SPSS (Statistical Package for the Social Sciences) 23.0 para Windows (SPSS Inc., Chicago, IL, USA). En primer lugar, se obtuvieron los datos descriptivos para los objetivos de medición del impacto económico, satisfacción y retorno de objetivos de patrocinio. Posteriormente se realizó el test Kolmogorov-Smirnov para realizar la prueba de normalidad de las variables métricas referentes a la evaluación de satisfacción. Según la distribución obtenida a través 
de esta prueba para las variables de impacto social y satisfacción $(p<0.05)$, se realizaron las pruebas no paramétricas K de Kruskall Wallis y U de Mann-Withney, permitiéndonos determinar si existían diferencias significativas entre medianas, y proporcionándonos un indicador comparativo de las medias de las distribuciones entre diferentes segmentos demográficos.

\section{Resultados}

\section{Impacto económico}

La Tabla 4 refleja los gastos, desglosados por conceptos, del total de 108 encuestados de fuera de Burgos. Con este análisis ACB se logra estimar el impacto económico directo del evento, que alcanza la cifra de 134,057.42 €.

Para calcular el importe del impacto indirecto se utilizaron las tablas Input-Output publicadas por la Junta de Castilla y León (2019). El impacto indirecto se calculó aplicando un multiplicador a cada uno de los gastos directos. El multiplicador es diferente para cada uno de los sectores de actividad. En nuestro caso el multiplicador medio final fue de 0.5025 , es decir por cada euro de gasto directo se produjo un efecto de arrastre indirecto de $0.5025 €$. El impacto indirecto estimado para la prueba fue de 73,866.83 $€$. De esta manera si se suma el impacto directo e indirecto, se estimó un impacto de 220,861.25€

A continuación, se muestra la Tabla 5 , en la que se observa el impacto económico indirecto. Se puede comprobar que los multiplicadores van desde 0.1680 para el sector de museos y exposiciones hasta 0.69 para el sector de alojamiento, que es uno de los sectores con mayor arrastre.

\section{Valoración y opinión del evento}

Para la valoración del evento se tuvieron en cuenta las 293 encuestas realizadas, tanto a participantes locales como de fuera de Burgos. En la Tabla 6 se muestran tanto los datos descriptivos como las comparaciones entre diferentes segmentos de poblaciones

Tabla 4. Resumen del impacto económico directo de la prueba.

\begin{tabular}{|c|c|c|c|c|c|c|c|c|c|}
\hline & Inscripción & $\begin{array}{l}\text { Aloja- } \\
\text { miento }\end{array}$ & $\begin{array}{c}\text { Restau- } \\
\text { ración }\end{array}$ & $\begin{array}{l}\text { Desplaza- } \\
\text { miento }\end{array}$ & $\begin{array}{c}\text { Compras } \\
\text { deportivas }\end{array}$ & $\begin{array}{l}\text { Otras } \\
\text { compras }\end{array}$ & $\begin{array}{l}\text { Visitas y } \\
\text { museos }\end{array}$ & $\begin{array}{c}\text { Otros } \\
\text { gastos }\end{array}$ & Total \\
\hline Media & 25.78 & 101.49 & 101.25 & 73.19 & 2.08 & 14.81 & 6.37 & 1.67 & 326.65 \\
\hline DS & 4.18 & 107,49 & 90.50 & 139.18 & 11.66 & 35.62 & 14.46 & 6.80 & \\
\hline Mediana & 23.43 & 75.00 & 90.00 & 40 & - & - & - & - & \\
\hline Moda & 23.43 & - & 100.00 & 40 & - & - & - & - & \\
\hline $\begin{array}{l}\text { Gasto } 108 \\
\text { foráneos }\end{array}$ & 2784.66 & 10,961 & 10,935 & 7905 & 225 & 1600 & 688 & 180 & \\
\hline $\begin{array}{l}\text { Gasto estimado } \\
450 \text { foráneos }\end{array}$ & $11,602.75$ & $45,670.83$ & $45,562.50$ & $32,937.50$ & 937,50 & 6666.67 & 2866.67 & 750 & $146,994.42$ \\
\hline $\begin{array}{l}\text { Impacto } \\
\text { económico } \\
\text { directo final }\end{array}$ & $11,602.75$ & $45,670.83$ & 45562.50 & $20,000(1)$ & 937.50 & 6666.67 & 2866.67 & 750 & $134,057.42$ \\
\hline
\end{tabular}

(1) El gasto estimado, que han realizado los corredores y acompañantes que han venido de fuera de Burgos a la prueba, ha ascendido a un total de 146,994 $€$. De ellos hay que estimar que una parte no se ha realizado en su totalidad dentro de Burgos. En concreto, los 32,937.5 € de gastos de desplazamiento se han realizado en su parte en el lugar de origen o durante el traslado. Una parte del repostaje de los vehículos (85\% de medio de transporte de los visitantes) se ha realizado en Burgos.

Tabla 5. Resumen del impacto económico indirecto de la prueba.

\begin{tabular}{|c|c|c|c|c|c|c|c|c|c|}
\hline & Inscripción & $\begin{array}{l}\text { Aloja- } \\
\text { miento }\end{array}$ & $\begin{array}{l}\text { Restau- } \\
\text { ración }\end{array}$ & $\begin{array}{l}\text { Desplaza- } \\
\text { miento }\end{array}$ & $\begin{array}{c}\text { Compras } \\
\text { deportivas }\end{array}$ & $\begin{array}{l}\text { Otras } \\
\text { compras }\end{array}$ & $\begin{array}{l}\text { Visitas y } \\
\text { museos }\end{array}$ & $\begin{array}{l}\text { Otros } \\
\text { gastos }\end{array}$ & Total \\
\hline $\begin{array}{l}\text { Impacto económico } \\
\text { directo }\end{array}$ & $11,602.75$ & $45,670.83$ & $45,562.50$ & 20,000 & 937.50 & 6666.67 & 2866.67 & 750 & $134,057.42$ \\
\hline $\begin{array}{l}\text { Multiplicador en tablas } \\
\text { Input-Output }\end{array}$ & 0.3721 & 0.6900 & 0.4629 & 0.3721 & 0.5167 & 0.5167 & 0.1680 & 0.3721 & 0.5025 \\
\hline $\begin{array}{l}\text { Impacto económico } \\
\text { indirecto }\end{array}$ & 4316.97 & $31,515.07$ & $21,090.65$ & $12,254.86$ & 484.36 & 3444.35 & 481.52 & 279.05 & $73,866.83$ \\
\hline $\begin{array}{l}\text { Total de impacto } \\
\text { económico } \\
\text { (directo+indirecto) }\end{array}$ & $15,919.72$ & $77,185.90$ & $66,653.15$ & $45,192.36$ & 1421.86 & $10,111.02$ & 3348.19 & 1029.05 & $220,861.25$ \\
\hline
\end{tabular}


Tabla 6. Datos descriptivos estudio de satisfacción-impacto social. Diferencias por género, franjas de edad y modalidad de participación.

\begin{tabular}{|c|c|c|c|c|c|c|c|c|c|}
\hline & & $\begin{array}{c}\text { Reconocimiento } \\
\text { para Burgos }\end{array}$ & $\begin{array}{l}\text { Orgullo para } \\
\text { la ciudad }\end{array}$ & $\begin{array}{c}\text { Perjudica } \\
\text { a otras } \\
\text { actividades }\end{array}$ & $\begin{array}{l}\text { Incrementa } \\
\text { el turismo }\end{array}$ & $\begin{array}{l}\text { Aumenta } \\
\text { el empleo }\end{array}$ & $\begin{array}{l}\text { El gasto Público } \\
\text { es necesario }\end{array}$ & $\begin{array}{l}\text { Recomendaría } \\
\text { Burgos }\end{array}$ & $\begin{array}{l}\text { Marca de } \\
\text { la ciudad }\end{array}$ \\
\hline \multirow[t]{2}{*}{ Total } & Media & 4.54 & 4.50 & 2.41 & 4.37 & 3.21 & 3.91 & 4.42 & 4.71 \\
\hline & DS & 0.69 & 0.76 & 1.29 & 0.87 & 1.22 & 1.15 & 0.80 & 0.56 \\
\hline \multirow{2}{*}{ Hombre } & Media & 4.56 & 4.49 & 2.47 & 4.37 & 3.22 & 3.92 & 4.42 & 4.71 \\
\hline & DS & 0.65 & 0.75 & 1.30 & 0.83 & 1.21 & 1.17 & 0.82 & 0.56 \\
\hline \multirow{2}{*}{ Mujer } & Media & 4.45 & 4.55 & 2.07 & 4.36 & 3.14 & 3.82 & 4.43 & 4.68 \\
\hline & DS & 0.87 & 0.79 & 1.14 & 1.08 & 1.25 & 1.04 & 0.69 & 0.56 \\
\hline \multirow{2}{*}{$18-29$} & Media & 4.61 & 4.53 & 2.26 & 4.29 & 3.11 & 3.71 & 4.34 & 4.76 \\
\hline & DS & 0.67 & 0.72 & 1.08 & 1.08 & 1.26 & 1.18 & 0.85 & 0.49 \\
\hline \multirow{2}{*}{$30-49$} & Media & 4.53 & 4.49 & 2.42 & 4.38 & 3.30 * & $4.02 *$ & 4.43 & 4.68 \\
\hline & DS & 0.67 & 0.75 & 1.32 & 0.80 & 1.21 & 1.08 & 0.77 & 0.56 \\
\hline \multirow[t]{2}{*}{$50-70$} & Media & 4.54 & 4.52 & 2.46 & 4.37 & $2.93 *$ & 3.61 * & 4.44 & 4.80 \\
\hline & DS & 0.77 & 0.82 & 1.29 & 0.96 & 1.18 & 1.31 & 0.88 & 0.626 \\
\hline \multirow[t]{2}{*}{ Maratón } & Media & 4.60 & 4.65 & 2.25 & 4.38 & 3.27 & 3.81 & 4.33 & 4.63 \\
\hline & DS & 0.53 & 0.55 & 1.28 & 0.91 & 1.22 & 1.23 & 0.73 & 0.52 \\
\hline \multirow[t]{2}{*}{$\begin{array}{l}\text { Media } \\
\text { maratón }\end{array}$} & Media & $4.50 *$ & 4.44 & 2.47 & 4.35 & 3.17 & 3.92 & 4.44 & 4.73 \\
\hline & DS & 0.72 & 0.81 & 1.24 & 0.88 & 1.20 & 1.14 & 0.80 & 0.58 \\
\hline \multirow[t]{2}{*}{ Relevos } & Media & $4.78^{*}$ & 4.70 & 2.13 & 4.48 & 3.43 & 4.04 & 4.43 & 4.70 \\
\hline & DS & 0.67 & 0.55 & 1.60 & 0.66 & 1.41 & 1.10 & 0.94 & 0.47 \\
\hline
\end{tabular}

* Existen diferencias a nivel de $p<0.05$.

Tabla 7. Porcentajes de identificación de las principales marcas patrocinadoras por parte de los participantes en función del recuerdo espontáneo e inducido.

\begin{tabular}{lcc}
\hline & $\begin{array}{c}\text { Recuerdo inducido } \\
\text { (El encuestado tiene total seguridad } \\
\text { de que es patrocinador del evento) }\end{array}$ & $\begin{array}{c}\text { Recuerdo espontáneo } \\
\text { (El encuestado menciona la marca al } \\
\text { preguntársele por patrocinadores del evento) }\end{array}$ \\
\hline Naming Sponsor & $277(94.5 \%)$ & $238(81.2 \%)$ \\
Sponsor técnico (calzado-ropa) & $236(80.5 \%)$ & $66(22.5 \%)$ \\
Entidad Financiera & $213(72.7 \%)$ & $64(21.8 \%)$ \\
Concesionario de automóviles & $144(49.1 \%)$ & $18(6.1 \%)$ \\
Universidad & $186(63.5 \%)$ & $18(6.1 \%)$ \\
Bebida azucarada & $101(34.5 \%)$ & $16(5.5 \%)$ \\
Polígono Industrial & $79(27 \%)$ & 0 \\
\hline
\end{tabular}

establecidos en función de género, edad o modalidad de participación. Se encontraron diferencias significativas $(p<0.05)$ en la percepción del reconocimiento para Burgos, siendo mayor en los corredores de relevos respecto a los de maratón. De la misma manera, la franja de edad media (30-49 años) percibe significativamente $(p<0.05)$ que el gasto público es necesario y que el evento es beneficioso para el empleo, respecto a la franja de edad superior (50-70 años).

\section{Recuerdo espontáneo e inducido de patrocinadores}

Para el recuerdo espontáneo e inducido de patrocinadores también se tuvieron en cuenta las 293 encuestas realizadas, tanto a participantes locales como de fuera de Burgos. La Tabla 7 muestra el resumen del recuerdo de marcas patrocinadoras, tanto de manera espontánea como de manera inducida. Se han sustituido las marcas identificadas por los encuestados por nombres genéricos para garantizar el anonimato de las empresas patrocinadoras.

\section{Discusión}

Los resultados obtenidos en las diferentes dimensiones analizadas, económica, social y publicitaria, son bastante positivos para todas las partes interesadas. Estos datos son de gran utilidad para las diferentes instituciones, ya que unos buenos resultados económicos y una generación positiva de la imagen del evento determina la intención de visitar en el futuro 
tanto la ciudad como el evento en concreto (Hallmann \& Wicker, 2012).

En el impacto económico, podemos observar que el resultado de impacto directo obtenido en el presente estudio $(134,057.42 €)$ es inferior al generado en otros eventos, como se muestra en la Tabla 1. Este hecho puede explicarse por varios factores. El primero de ellos es que, en los demás casos, el número de participantes fue mayor, aumentando en consecuencia la participación de corredores foráneos, e incrementando el impacto directo. En segundo lugar, hay territorios que exigen unos mayores costes de participación, como las islas Baleares y Canarias, generando un impacto directo más elevado (RTVC, 2017; SánchezVenzalá, 2019). Finalmente, se ha de considerar que la práctica más habitual es extrapolar el gasto medio al número de participantes, en lugar de aplicar estudios de campo con encuestas reales a los asistentes, como sí se ha realizado en el presente estudio. Por esta razón, para obtener resultados más cercanos a la realidad se recomienda siempre realizar un cuestionario que identifique el perfil de asistente y recoja datos sobre los gastos en alojamiento, comida y bebida, transporte y ocio en el municipio donde se celebra el evento. Asimismo, es prioritario evitar fallos metodológicos como la inclusión del público local, los time-switchers o los asistentes casuales, cuya visita a la ciudad no ha sido consecuencia del evento deportivo, lo cual convertiría los resultados de impacto económico en más optimistas (Barajas et al., 2016).

En línea con lo anterior, podemos observar que el gasto medio de corredores foráneos en el Maratón de Burgos (326.65 €) es semejante al de otras ciudades como Bilbao (298.50€) o Vitoria (372.54€), pero dista mucho de ciudades como Valencia (960.41 €) o Gran Canaria (2145.92 €) (Tabla 1). Al comparar los resultados obtenidos con otros maratones internacionales, nuestras cifras se sitúan por debajo de las recogidas por Wicker y Hallmann (2013), quienes obtuvieron una predisposición de gasto de $568 €$ para pruebas en Europa y $1429 €$ para eventos en América. Parece evidente que aspectos externos, como el carácter internacional de la prueba, la disponibilidad de alojamiento y los precios medios de los servicios de hostelería, afecten de manera diferente en función de los territorios. Finalmente, en lo referente al impacto total (directo + indirecto), la cifra obtenida muestra coherencia otros eventos similares, como recoge la Tabla 1.

Respecto a los resultados de impacto social y satisfacción, se observa que los participantes perciben el Maratón de Burgos 2019 de manera bastante positiva en líneas generales, incluso con valoraciones superiores que estudios anteriores aplicados a eventos de maratón (Parra-Camacho et al., 2020b) o a otras modalidades deportivas, como Fórmula 1 (Añó et al. 2012). Por su parte, los resultados no arrojan grandes diferencias entre la mayoría de grupos de población analizados, aunque hubo diferencias significativas en función de la edad. A este respecto, los corredores de mediana edad (30-49 años) se mostraron más optimistas respecto a la generación de empleo y a la necesidad de gasto público en comparación con los corredores mayores de 50 años. Estas diferencias pueden ser debidas a los diferentes momentos de la vida laboral en los que se encuentran sendos grupos de edad, más que a elementos puramente deportivos. En este sentido, tal y como indican Parra-Camacho et al. (2015), estas opiniones desfavorables pueden deberse al elevado gasto realizado en ciudades con un contexto socioeconómico desfavorable. Por su parte, los datos de satisfacción y recomendación de la ciudad anfitriona coinciden con otros estudios donde se han analizado eventos deportivos con una alta participación (Burillo et al., 2018). Tal y como afirma Blanco-Gregory (2018), la actividad deportiva contribuye a fomentar una imagen positiva de un destino turístico, algo de lo que se favorece la ciudad de Burgos.

Finalmente, respecto al estudio de retorno de objetivo de patrocinio, los resultados favorecen claramente al patrocinador principal, cuyo nombre consta en la denominación del evento. Por ende, su recuerdo espontáneo e inducido es el mayor de todos. No sucede así con el resto de marcas, cuyo recuerdo espontáneo baja considerablemente, por debajo del 50\%. Esta misma situación también se observa en otros eventos de running, donde el espónsor principal obtuvo resultados parecidos, y el resto de marcas un recuerdo espontáneo más bajo (De la Riva et al., 2017). La importancia de estos datos yace en la necesidad de estimular la atención de los participantes a través de medios, bien sean impresos, como carteles o marquesinas; o digitales, como las redes sociales (Alonso-Dos-Santos et al., 2019).

\section{Conclusiones}

Las conclusiones que responden los objetivos de investigación son:

- El gasto directo estimado de corredores y acompañantes foráneos a Burgos ascendió a 134,057.42 €.

- La estimación del gasto indirecto del evento alcanzó los 73,866.83 € extraídos tras la utilización de las tablas Input-Output.

- El patrocinador principal presenta una visibilidad claramente mayor, que se traduce en un recuerdo inducido $(94.5 \%)$ y espontáneo (81.2\%) muy su- 
perior al del resto de marcas participantes (menor del 50\%).

- La opinión relativa a la satisfacción, impacto social e impacto turístico de la carrera fue similar en la mayoría de diferentes grupos analizados. Los participantes de edades medias (30-49 años) mostraron mayor satisfacción con la inversión realizada y el empleo generado.

Nuestros resultados tienen importantes implicaciones prácticas, principalmente para los organizadores de eventos deportivos en tres direcciones: permitiendo acertar en la toma de decisiones para asegurar la continuidad del evento, adaptar las campañas de promoción y favorecer la identificación de fortalezas y debilidades del evento.

Entre las principales limitaciones, se encuentra el alto error muestral que nos encontramos en los corredores foráneos, que debería haber sido inferior al 5\%.
Esto pudo ser debido a que el día de la recogida de dorsales, el sábado 12 de octubre de 2019, era festivo, por lo que muchos comercios estaban cerrados. Este hecho fue mencionado por muchos de los encuestados. Asimismo, el cuestionario ad-hoc elaborado para la satisfacción e impacto social podría ser validado en todos sus ítems, elemento que se tendrá en cuenta para futuras publicaciones.

Futuras líneas de investigación podrían abordar el estudio de eventos deportivos tras la interrupción producida por la COVID-19, explicando cuál es la percepción de los asistentes y cómo ha afectado a nivel económico.

\section{Agradecimientos}

Este estudio se realizó con la colaboración de un equipo de encuestadores de la Universidad Isabel I, que fue colaboradora del evento y con la autorización y ayuda de los organizadores del mismo. 
Anexo 1. Encuesta para corredores participantes

\section{Género}

$\square$ Hombre

$\square$ Mujer

$\begin{array}{lr}\text { Año de nacimiento __ Tu respuesta } \\ \text { Código Postal } & \text { Tu respuesta }\end{array}$

\section{Participante en:}

$\square$ Maratón

$\square$ Media Maratón

$\square$ Relevos $42 \mathrm{~K} / 4$

Número de dorsal Tu respuesta

Estimación de su gasto asociado al Maratón (incluir el de los acompañantes)

A continuación, deberá indicar el total de $€$ gastados en diferentes conceptos.

Gastos en alojamiento en Burgos Tu respuesta

Gasto en comida y bebida en Burgos Tu respuesta

Gasto en el desplazamiento

(para llegar a Burgos y dentro de la ciudad) Tu respuesta

Gasto en compra de material deportivo (en Burgos) Tu respuesta

Gasto en otras compras (en Burgos) Tu respuesta

Gasto en visitas y museos (en Burgos) Tu respuesta

Otros gastos (en Burgos) Tu respuesta

¿Qué marcas principales patrocinan este maratón?

¿Podría decirme alguna? Tu respuesta

¿Son patrocinadores del Maratón de Burgos?

\begin{tabular}{|c|c|c|c|c|c|}
\hline & Sí, seguro & Creo que sí & No lo sé & Creo que no & No, seguro \\
\hline \multicolumn{6}{|c|}{ Coca Cola } \\
\hline \multicolumn{6}{|l|}{ Adidas } \\
\hline \multicolumn{6}{|l|}{ Nike } \\
\hline \multicolumn{6}{|c|}{ Campofrío } \\
\hline \multicolumn{6}{|l|}{ El Pozo } \\
\hline \multicolumn{6}{|c|}{ Bankia Laboral Kutxa } \\
\hline \multicolumn{6}{|c|}{ Autovican Renault } \\
\hline \multicolumn{6}{|c|}{ Asociación Polígono Industrial Burgos Este } \\
\hline \multicolumn{6}{|c|}{ San Pablo Burgos } \\
\hline \multicolumn{6}{|c|}{ Universidad Isabel I } \\
\hline Mizuno & & & & & \\
\hline
\end{tabular}




\section{Opinión del evento}

\begin{tabular}{|c|c|c|c|c|c|}
\hline & $\begin{array}{l}\text { Totalmente en } \\
\text { desacuerdo }\end{array}$ & En desacuerdo & Indiferente & De acuerdo & $\begin{array}{l}\text { Totalmente } \\
\text { de acuerdo }\end{array}$ \\
\hline \multicolumn{6}{|l|}{$\begin{array}{l}\text { Aumenta el reconocimiento y } \\
\text { promoción de Burgos }\end{array}$} \\
\hline \multicolumn{6}{|l|}{$\begin{array}{l}\text { Representa un elemento de orgullo } \\
\text { para la ciudad de Burgos }\end{array}$} \\
\hline \multicolumn{6}{|l|}{$\begin{array}{l}\text { Altera el ritmo habitual y perjudica } \\
\text { otras actividades }\end{array}$} \\
\hline \multicolumn{6}{|l|}{$\begin{array}{l}\text { Provoca un incremento del turismo } \\
\text { durante y después del evento }\end{array}$} \\
\hline \multicolumn{6}{|l|}{ Aumenta el empleo } \\
\hline \multicolumn{6}{|l|}{$\begin{array}{l}\text { El gasto público es necesario para la } \\
\text { celebración de este tipo de eventos }\end{array}$} \\
\hline \multicolumn{6}{|l|}{$\begin{array}{l}\text { Hace que mucha gente quiera volver a } \\
\text { la ciudad para conocerla mejor }\end{array}$} \\
\hline $\begin{array}{l}\text { Es una oportunidad para aumentar } \\
\text { la marca de Burgos como ciudad } \\
\text { relacionada con el deporte }\end{array}$ & & & & & \\
\hline
\end{tabular}

¿Reside en Burgos?

$\square$ Sí

$\square$ No

Comentarios adicionales 


\section{BIBLIOGRAFÍA}

42K Running. (2016). Gran retorno económico para las ciudades que albergan eventos deportivos. Recuperado 25 de febrero de 2020, de https://42krunning.com/24177/

Agha, N., \& Rascher, D. A. (2016). An explanation of economic impact: Why positive impacts can exist for smaller sports. Sport, Business and Management: An International Journal, 6(2), 182-204. https://doi. org/10.1108/SBM-07-2013-0020

Aguilera, F. (2017). La participación en el Marató Costa Daurada Tarragona 2017 crece un 50\% en un año. Recuperado 25 de febrero de 2020, de http://www.wangconnection.com/la-participacion-en-el-maratocosta-daurada-tarragona-2017-crece-un-50-en-un-ano/

Alonso-Dos-Santos, M., Calabuig-Moreno, F., \& Crespo-Hervás, J. (2019). Influence of perceived and effective congruence on recall and purchase intention in sponsored printed sports advertising: An eye-tracking application. International Journal of Sports Marketing and Sponsorship, 20(4), 617-633. https://doi.org/10.1108/IJSMS-10-2018-0099

Andalucía Información. (2016). El Ayuntamiento cifra en 9,4 millones el impacto de la Maratón 2016, un 20\% más que en 2015. Recuperado 4 de marzo de 2020, de https://andaluciainformacion.es/malaga/579150/ el-ayuntamiento-cifra-en-94-millones-el-impacto-de-la-maraton2016-un-20-mas-que-en-2015/

Añó, V., Calabuig-Moreno, F., \& Parra-Camacho, D. (2012). Impacto social de un gran evento deportivo: el Gran Premio de Europa de Fórmula 1. Cultura, Ciencia y Deporte, 7(19), 53-65.

Banks, A. (1992). How to access new place-based media. Advertising Age, 11(30), 36.

Baños, J., Pujol, F., \& Rodríguez, P. (2012). Análisis del impacto económico de la celebración del Campeonato del Mundo de Patinaje de Velocidad. Estudios de Economía Aplicada 30(2), 703-731. https://doi. org/10.6018/254101

Barajas, Á., Coates, D., \& Sánchez Fernández, P. (2016). Beyond retrospective assessment. Sport event economic impact studies as a management tool for informing event organization. European Research on Management and Business Economics, 22(3), 124-130. https://doi. org/10.1016/j.iedee.2015.05.001

Blanco-Gregory, R. (2018). La influencia de los eventos deportivos como motivación en la elección de un destino turístico y como motor de desarrollo del mismo. Eracle. Journal of Sport and Social Sciences, 1(1), 55-67. https://doi.org/10.6093/2611-6693/5778

Bosch, J., Murillo, C., \& Raya, J. M. (2019). La importancia económica del sector deportivo y el impacto económico de los eventos deportivos. Papeles de Economía Española, Deporte y Economía, 159(abril), 261-274.

Burillo, P., De la Riva, L. E., García-Unanue, J., Sánchez-Sánchez, J., Felipe, J. L., \& Fernández-Luna, A. (2018). Spectator spending and satisfaction analysis in the 2017 Madrid Tennis Open according to the attendee profile. Journal of Physical Education and Sport, 18(3), 12711275. https://doi.org/10.7752/jpes.2018.s3189

Burillo, P., \& Pérez-González, B. (2016). Análisis del comportamiento de los consumidores ante el factor precio en el mercado de calzado para corredores. SPORT TK: Revista EuroAmericana de Ciencias Del Deporte, 5(1), 163-168. https://doi.org/10.6018/254171

Calabuig-Moreno, F., Crespo-Hervas, J., Nunez-Pomar, J., Valantinè, I., \& Staškevičiūtè-Butienè, I. (2016). Role of perceived value and emotions in the satisfaction and future intentions of spectators in sporting events. Inžinerine ekonomika, 27(2), 221-229.

Clough, P., Shepherd, J., \& Maughan, R. (1989). Motives for Participation in Recreational Running. Journal of Leisure Research, 21(4), 297309. https://doi.org/10.1080/00222216.1989.11969806

CMD Sport (2019). El running, el deporte que más patrocinios atrae. Recuperado el 25 de febrero de 2020, de https://www.cmdsport.com/ esencial/cmd-running/running-deporte-mas-patrocinios-atrae/

Cobb, S., \& Olberding, D. J. (2007). The Importance of Import Substitution in Marathon. International Journal of Sport Finance, 2(2), 108-118.

Coleman, R., \& Ramchandani, G. (2010). The hidden benefits of nonelite mass participation sports events: an economic perspective. International Journal of Sports Marketing and Sponsorship, 12(1), 19-31. https://doi.org/doi:10.1108/IJSMS-12-01-2010-B004
COPE.(2017).ElMaratón BP Castellón dejaunimpactoeconómico de 1.600.000. Recuperado 25 de febrero de 2020, de https://www.cope.es/emisoras/ comunidad-valenciana/castellon-provincia/castellon/noticias/maraton-castellon-deja-impacto-economico-1600000-20170223_37099

Crompton, J. L. (1995). Economic Impact Analysis of Sports Facilities and Events: Eleven Sources of Misapplication. Journal of Sport Management, 9(1), 14-35. https://doi.org/10.1123/jsm.9.1.14

Crompton, J. L. (2006). Economic Impact Studies: Instruments for Political Shenanigans? Journal of Travel Research, 45(1), 67-82. https:// doi.org/10.1177/0047287506288870

De la Riva L. E., Cavalli, F., Sánchez-Sánchez, J., García-Unanue, J. F., Felipe, J. L., Burillo , P., \& Fernández-Luna, Á. (2017). Notoriedad de marcas patrocinadoras en eventos deportivos: Asociación indirecta de marcas. Recuperado el 29 de octubre de 2020, de http://hdl.handle. net/11268/6762

Duclos-Bastías, D., Vallejo-Reyes, F., Giakoni-Ramírez, F., \& Parra-Camacho, D. (2021). Validation of the Marathon Motivation Scale in Chile. Journal of Human Sport and Exercise, 16(12), en prensa. En prensa. https://doi.org/10.14198/jhse.2021.162.10

El Correo. (2019). La maratón nocturna traerá a Bilbao a 13.400 atletas de más de 50 países. Recuperado 25 de febrero de 2020, de https://www. elcorreo.com/deportes/mas-deportes/maraton-nocturna-traera20191010111315-nt.html?ref=https:\%2F\%2Fwww.google.com\%2F

Europa Press. (2019). El Maratón de Madrid «más artístico» tendrá un impacto de casi 43 millones. Recuperado el 25 de febrero de 2020 de https://www.europapress.es/deportes/noticia-maraton-madrid-masartistico-tendra-impacto-casi-43-millones-20190410153124.html

Felipe, J. L., Burillo, P., De la Riva, L. E., Fernández-Luna, Á., PérezGonzález, B., \& García-Unanue, J. (2018). Analysis of annual expenditure in equipment by $5 \mathrm{k}$ and $10 \mathrm{k}$ runners according to race time. Journal of Physical Education and Sport, 18(3), 1330-1334. https://doi. org/10.7752/jpes.2018.s3197

Felipe, J. L., Fernández-Luna, Á., Ubago-Guisado, E., Gómez-González, C., \& Colino, E. (2015). El gasto de los espectadores durante el Mundobasket 2014 y su intención de recomendar el país organizador. En A. DíazSuárez y R. Ibáñez-Pérez (Coords.), La importancia del impacto económico del deporte en la sociedad actual (pp. 13-17). Universidad de Murcia.

Fernández, P., \& Alonso, Á. (2012). Los Eventos Deportivos Como Generadores De Impacto Económico: Factores Clave y Medición. Webs. Uvigo.Es.

Fourie, J., \& Santana-Gallego, M. (2011). The impact of mega-sport events on tourist arrivals. Tourism Management, 32(6), 1364-1370. https://doi.org/https://doi.org/10.1016/j.tourman.2011.01.011

Funk, D. C., Toohey, K., \& Bruun, T. (2007). International Sport Event Participation: Prior Sport Involvement; Destination Image; and Travel Motives. European Sport Management Quarterly, 7(3), 227-248. https://doi.org/10.1080/16184740701511011

Gibson, H. J., Kaplanidou, K., \& Kang, S. J. (2012). Small-scale event sport tourism: A case study in sustainable tourism. Sport Management Review, 15(2), 160-170. https://doi.org/https://doi.org/10.1016/j. smr.2011.08.013

Hallmann, K., Kaplanidou, K., \& Breuer, C. (2011). Event image perceptions among active and passive sports tourists at marathon races. International Journal of Sports Marketing and Sponsorship, 12(1), 32-47. https://doi.org/10.1108/ijsms-12-01-2010-b005

Hallmann, K., \& Wicker, P. (2012). Consumer profiles of runners at marathon races. International Journal of Event and Festival Management, 3(2), 171-187. https://doi.org/10.1108/17582951211229717

Jago, L., \& Dwyer, L. (2006). Economic evaluation of special events: a practitioner's guide. Altona, Victoria: Common Ground Publishing.

Junta de Castilla y León. (2019). Tablas Input-Output 2010. Recuperado el 25 de febrero de 2020, de http://www.jcyl.es/web/jcyl/Estadistica/ es/Plantilla100/1284165712696/1246890364336/_/_

Kaplanidou, K. K., Jordan, J. S., Funk, D., \& Rindinger, L. L. (2012). Recurring sport events and destination image perceptions: Impact on active sport tourist behavioral intentions and place attachment. Journal of Sport Management, 26(3), https://doi.org/10.1123/jsm.26.3.237 
Lancelot Digital. (2015). El Maratón Internacional dejó un impacto económico de más de 665.000 euros en la isla. Recuperado el 25 de febrero de 2020, de https://www.lancelotdigital.com/vida-social/el-maraton-internacionaldejo-un-impacto-economico-de-mas-de-665-000-euros-en-la-isla

Li, S. N., \& Jago, L. (2013). Evaluating economic impacts of major sports events - A meta analysis of the key trends. Current Issues in Tourism, 16(6), 591-611. https://doi.org/10.1080/13683500.2012.736482

Martínez-Cevallos, D., Alguacil, M., \& Calabuig-Moreno, F. (2020a). Influence of Brand Image of a Sports Event on the Recommendation of Its Participants. Sustainability, 12(12), 5040. https://doi. org/10.3390/su12125040

Martínez-Cevallos, D., Proaño-Grijalva, A., Alguacil, M., Duclos-Bastías, D., \& Parra-Camacho, D. (2020b). Segmentation of Participants in a Sports Event Using Cluster Analysis. Sustainability, 12(14), 5641. https://doi.org/10.3390/su12145641

Maudos, J., Aldás, J., Benagues, E., \& Zaera, I. (2020). 39 Maratón Valencia Trinidad Alonso EDP 2019: Impacto económico y valoración de los corredores.

Ministerio de Cultura y Deporte. (2020). Anuario de Estadísticas Deportivas 2020. Madrid: Consejo Superior de Deportes.

Murillo, C., Carles, M., Llop, M., Moya, X., \& Planas, D. (2016). Campeonato Mundial de Patinaje Artístico Reus 2014: opinión de los participantes y legado económico. SPORT TK-Revista EuroAmericana De Ciencias Del Deporte 5(1), 107-118. https://doi.org/10.6018/254101

Murua, J. (2013). Eventos deportivos: economía real o Monopoly. Economía en chándal. Recuperado 25 de febrero de 2020, de https://economiaenchandal.com/2013/09/23/los-eventos-deportivos-economiareal-o-monopoly/

Ogles, B., \& Masters, K. (1995). Obligatory Running and Gender: A «An Analysis of Participative Motives and Training Habits. International Journal of Sport Psychology, 26(2), 233-248

Parra-Camacho, D., Aguado-Berenguer, S., \& Núñez-Pomar, J. (2015). Los costes de la celebración de un evento deportivo: percepción de la comunidad de acogida. Journal of Sports Economics \& Management, 5(1), 17-36.

Parra-Camacho, D., Alonso-Dos-Santos, M., \& González-Serrano, M H. (2020a). Amateur runners' commitment: an analysis of sociodemographic and sports habit profiles. International Journal of Environmental Research and Public Health, 17(3), 925. doi: https://doi. org/10.3390/ijerph17030925

Parra-Camacho, D., González-García, R. J., \& Alonso-Dos-Santos, M. (2020b). Social impact of a participative small-scale sporting event. Sport, Business and Management, ahead-of-print. https://doi. org/10.1108/SBM-12-2019-0119

Paz, C., Vázquez, R., \& Santos, L. (2000). Publicidad y eficacia publicitaria: Influencia de la posición, repetición y estilos publicitarios en la eficacia de los anuncios televisivos entre jóvenes. Facultad de Ciencias Económicas y Empresariales, Universidad de Oviedo.
Pérez-Acosta, A. M. (2006). Prueba empírica de un modelo asociativo de recuerdo de marcas publicitarias con datos de mercado real. Suma Psicológica, 13(1), 85-95

RTVC. (2017). Tres millones de euros de impacto económico de la Gran Canaria Maratón. Recuperado el 25 de febrero de 2020, de http://www. rtvc.es/deportes/tres-millones-de-euros-de-impacto-economico-dela-gran-canaria-maraton-163728.aspx\#.XlWUpGhKhhF

Saaymann, M., \& Saayman, A. (2012). The economic impact of the Comrades Marathon. International Journal of Event and Festival Management, 3(3), 220-235. https://doi.org/10.1108/17582951211262675

Sáez-Vegas, L., Mediano-Serrano, L., \& Elizagarate-Gutiérrez, V. (2011) Creación y desarrollo de marca ciudad, análisis de los registros de marca de las principales ciudades españolas. Revista de dirección y administración de empresas, (18), 125-156.

Salgado-Barandela, J., Barajas, Á., Lera-López, F., \& Sánchez-Fernández, P. (2013). Impacto económico de los eventos deportivos: modelo y praxis. Revista Intercontinental de Gestão Desportiva, 3(1), 38-50.

Salguero, A., \& Martos, P. (2011). Desigualdad de género en competiciones populares de fondo. Apunts. Educación Física y Deportes, 1(103), 91-100.

Sánchez-Fernández, P., \& Rodríguez-Campo, M. L. (2012). Los Eventos deportivos como acción estratégica del marketing de ciudades: el caso de Galicia. Revista Intercontinental de Gestão Desportiva, 2(1), 16-29.

Sánchez-Fernández, P., Salgado-Barandela, J., Rodríguez-Rodríguez, A., \& Barajas, Á. (2016). Impacto económico del XXI Campeonato de España "Open" de Invierno Master de Natación de Pontevedra 2015. SPORT TK-Revista EuroAmericana De Ciencias Del Deporte,5(1), 169180. https://doi.org/10.6018/254191

Sánchez-Venzalá, T. (2019). El deporte, inyección económica. Recuperado 25 de febrero de 2020, de https:/www.periodicodeibiza.es/deportes/ deporte-ibiza/2019/05/10/1078925/deporte-inyeccion-economica. html

Theodorakis, N. D., Kaplanidou, K., \& Karabaxoglou, I. (2015). Effect of Event Service Quality and Satisfaction on Happiness Among Runners of a Recurring Sport Event. Leisure Sciences, 37(1), 87-107. https:// doi.org/10.1080/01490400.2014.938846

Wicker, P., \& Hallmann, K. (2013). Estimating consumer's willingnessto-pay for participation in and traveling to marathon events. Event Management, 17(3), 271-282. https://doi.org/10.3727/15259951 3X13708863377953

Vegara-Ferri, J. M., Carboneros, M., \& Angosto, S. (2020). Percepción de calidad, impacto sociocultural, imagen de destino e intenciones futuras del turista participante en un evento náutico sostenible. Cultura, Ciencia y Deporte. En prensa.

Zurich Maratón de Sevilla. (2020). La nueva dimensión del Zurich Maratón de Sevilla. Recuperado 25 de febrero de 2020, de https://www.zurichmaratonsevilla.es/web-noticia/leer/nueva-dimension 